\title{
Records Auditing to Assess Nursing Functions in Intensive Care Unit at Main Assiut University Hospital
}

\author{
Fatma Rushdy Mohamed \& Samah Mohamed Abdalla \\ Assistant Professor of Nursing Administration, Faculty of Nursing, Assiut University, Egypt. \\ E-mail of the corresponding author: drfatmarushdy@yahoo.com
}

\begin{abstract}
Clinical audit is one of the main tools to establish whether the best evidence is being used in practice, as it identifies any gaps between what is done and what should be done, and rectifies any deficiencies in the actual processes of care. Clinical audit has been increasingly required for the accreditation process in every modern healthcare system. Aim of the study: Record auditing to assess nursing functions in general Intensive Care Unit at main Assiut University Hospital. Subject and method: A descriptive research design was utilized in the present study. The study sample constituted of 50 patients' records (medical and nursing records). Study tool: Auditing of Nursing Functions questionnaire consisted of two parts: $1^{\text {st }}$ part included two questions related to socio-demographic data of patient includes: (name of patient, hospital number); $2^{\text {nd }}$ part of the questionnaire adopted from (Phaneuf's nursing audit, 1955) which includes fifty items of nursing care functions. Results: Illustrated that none of the study subject had an excellent auditing of nursing and medical care, nearly two thirds of the data audited were good, more than one quarter were incomplete in auditing of nursing and medical care; and eight percent had a poor auditing of nursing and medical care. Recommendations: In- service training program for nurses needed to improve the quality of service. Replicate of these study at different units in health care settings to ensure effectiveness of the tool and serving as basis for planning new programs; identification of areas of strength and weakness in various settings.
\end{abstract}

\section{Key Words: Record Auditing, Nursing Functions \& Intensive Care Unit.}

\section{Introduction}

Clinical audit has a history reaching back to the work of Florence Nightingale in the 1859 s, where she used an epidemiological method of review for monitoring rates of nosocomial infections in relation to standards of hygiene. Health services are now utilizing audit as an integral part of their quality improvement strategies and accreditation processes (Centre for Clinical Governance Research in Health, 2009).

Healthcare audit is not new. It is a quality improvement activity that most healthcare employees have done for a long time as part of everyday practice. The purpose of healthcare audit is to monitor to what degree standards for any given healthcare activity are met, identify reasons why they are not met, and identify and implement changes to practice to meet those standards. It is the duty of all clinicians to ensure that they deliver the best care to their patients. All clinicians should be auditing their work. Clinicians have a duty to use the findings of audit to improve clinical care and move towards best practice i.e. audit is an essential tool for Continuous Quality Improvement (CQI) (Majella, 2008).

Clinical audit is a broad term which encompasses several of the other quality improvement strategies reviewed in this series including record reviews, peer review, standard reviews (to see if standards are being met, guidelines followed and / or evidence based practice utilized) and patient satisfaction surveys. The purpose of clinical audits is essentially to improve the quality of healthcare services by systematically reviewing the care provided against set criteria. The gap between the criteria and the assessed performance provides guidance for priority improvement strategies (Novo et al., 2006).

The word "Audit" today is specifically concerned with the checking and endorsing of financial accounts. It is a control to improve the quality of patient care. Auditing is done on some expected set standards. An audit is a systematic data collection process that commonly focuses on documentation, audits in regard to patient care are conducted by examining patient care records when records are audited. It is assumed that action not documented did not occur when patient care demands are high (Walker, 2006).

Nursing Audit is an important component of medical audit. Increase in the public awareness of their rights of safety and high cost of medical treatment necessitate that the nurses should become more accountable for care they deliver. Hence the nursing process has become a legal document in many countries (Hysong et al., 2006).

According to Elison, "Nursing audit refers to assessment of the quality of clinical nursing". According to Goster Walfer, (i) Nursing audit is an exercise to find out whether good nursing practices 
are followed and (ii) The audit is a means by which nurses by themselves can define standards from their point of view and describe the actual practice of nursing. Nursing audit is the process of analyzing data about the nursing process of patient's outcome to evaluate the effectiveness of nursing intervention (Reena \& Indarjit, 2011).

Diana Sale, (2005) mentioned that there are seven functions of professional nursing care which used as the framework for an audit. These functions are: $1^{\text {st }}$ Nursing care of the patient, $2^{\text {nd }}$ Care given by other professionals, $3^{\text {rd }}$ Observation of signs, symptoms and reaction, $4^{\text {th }}$ Application and execution of nursing procedures and techniques, $5^{\text {th }}$ Promotion of physical and emotional health by direction and teaching, $6^{\text {th }}$ Reporting and recording, and finally, Application and execution of physician's legal orders.

\section{Significance of the study}

The evidence base for clinical audit is increasing. Lack of evidence may stem from two major sources: the difficulty in comparing results across studies (because of differences in settings, participants, foci of intervention and the interventions themselves vary) and because implementation of audit and feedback strategies may themselves be subject to serious limitations and barriers. As with all forms of clinical review, the effectiveness, efficiency and usefulness of clinical audit appears to depend heavily on the sponsoring institutions ability to support and facilitate the audit, feedback and, most importantly, improvement processes required to complete the audit loop.

\section{Aim of the study}

Record auditing to assess nursing functions in general Intensive Care Unit at main Assiut University Hospital.

Research hypotheses

Functions of observations of symptoms and reactions, application and execution of nursing procedures and techniques, promotion of physical and emotional health by direction and teaching, and supervision of patient will be highest in mean scores than functions of application and execution of physician's legal orders, supervision of those participating in care, and reporting \& recording.

\section{Subject and Method}

Study design

A descriptive research design was utilized in the present study.

\section{I-Setting}

This study was conducted at General Intensive Care Unit which contains two rooms with bed capacity of eight beds in Main Assiut University Hospital.

\section{II-Subjects}

The study sample constituted of 50 patients' records (medical and nursing records) are reviewed immediately after documenting the care in the patient records. The number of study subjects was detected by using Schlescelman,(1982) formula to calculate study subjects were required .

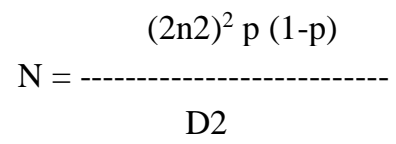

\section{Where}

$\mathrm{N}=$ sample size

$\mathrm{P}=0.50$

$\mathrm{D}=0.50 \times 10 \%=0.005$

III- Tool of the study

Auditing of Nursing Functions questionnaire consisted of two parts:

- $1^{\text {st }}$ part included two questions related to sociodemographic data of patient includes: (name of patient, hospital number).

- $2^{\text {nd }}$ part of the questionnaire adopted from (Phaneuf's nursing audit, 1955) cited by (Diana Sale, 2005) included of fifty items which consider functions of nursing care, it divided into seven functions: $1^{\text {st }}$ was related to Application and execution of physician's legal orders which contains six items. The possible responses of this factor were: 7 for yes and zero for no, with total score for this factor (42); $2^{\text {nd }}$ was related to Observations of symptoms and reactions which contain six items. The possible responses of this factor were: 7 for yes and zero for no, with total score for this factor $(42) ; 3^{\text {rd }}$ was related to Supervision of patient which contains seven items. The possible responses of this factor were: 4 for yes and zero for no, with total score for this factor (28); $4^{\text {th }}$ was related to Supervision of those participating in care (except the physician) which contains four items. The possible responses of this factor were: 5 for yes and zero for no, with total score for this factor $(20) ; 5^{\text {th }}$ was related to Reporting and recording which contains five items. The possible responses of this factor were: 4 for yes and zero for no, with total score for this factor (20). $6^{\text {th }}$ which related to Application and execution of nursing procedures and techniques which contains sixteen items The possible responses of this factor were: 2 for yes and zero for no, with total score for this factor (32); and $7^{\text {th }}$ was related to Promotion of physical and emotional health by direction and teaching which contains six items. The possible responses of this factor were: 3 for yes, and zero for no, with total score for this factor (18). the total score of the questionnaire is obtained by multiplying the total of the 
individual component scores by its value as follows: 161 to 200 considered excellent in performing nursing care,160-121 considered good, 120 to 81 was incomplete, 80 to 41 was poor, and 40 to zero was unsafe care.

\section{Administrative Design}

An official permission was obtained from the Director of main Assiut University Hospital, Head of General Intensive Care Unit.

V. Operational Design

This design explains the steps of actual implementation of the study, including the pilot and the field work.

\section{Pilot study}

A pilot study was conducted to test both the clarity and understandability of the items and detect the obstacles and problems that may be encountered during data collection. It also helped to estimate time needed to fill questionnaire. It was carried out on 10 patient charts from Neurosurgery Intensive Care Unit at main Assiut University Hospital by reviewing the auditing of these care in the patient records every record takes about half hour in each shift the total period for collection of data in the pilot study takes about 15 days. They excluded from the total study sample because the study done in General Intensive Care Unit based on the result of the pilot study there is no modifications were done.

\section{Fieldwork}

After the finalization of the study tool, the actual data collection was started in June 2013 and ended August 2013. The researchers met with the eligible participated nurses, explained to them the purpose of the study, and asked for their oral consent to participate then the researchers reviewing the care documented on the patient chart. The time taken for every questionnaire to be completed during morning shifts.

\section{Ethical considerations}

All the relevant principles of ethics in research were followed. The study protocol was approved by the pertinent authority. Participants' consent to participate was obtained after informing them about their rights to participate, refuse, or withdraw at any time. Total confidentiality of any obtained information was ensured. The study maneuver could not entail any harmful effects on participants.

\section{Statistical Design}

Upon completion of data collection, data entry was done using Epi-Info 6.04 computer software package, while statistical analysis was done using SPSS 14.0 statistical software packages. Data were presented using descriptive statistics in the form of frequencies and percentages. Total score were calculated and percentage of each score was done.

\section{Vol (2), No (3), Supplement June 2014}




\section{Results}

Table (1): Distribution of auditing seven nursing functions at Intensive care Unit in Main Assiut University Hospital.

\begin{tabular}{|c|c|c|c|c|}
\hline \multirow{2}{*}{ Items } & \multicolumn{2}{|c|}{ Yes } & \multicolumn{2}{|c|}{ No } \\
\hline & No. & $\%$ & No. & $\%$ \\
\hline \multicolumn{5}{|l|}{ Application and execution of physician's legal orders } \\
\hline Medical diagnosis complete & 20 & 40.0 & 30 & 60.0 \\
\hline Orders complete & 48 & 96.0 & 2 & 4.0 \\
\hline Orders current & 42 & 84.0 & 8 & 16.0 \\
\hline Orders promptly executed & 38 & 70.0 & 12 & 30.0 \\
\hline Evidence that nurse understood cause and effect & 22 & 44.0 & 28 & 56.0 \\
\hline Evidence that nurse took health history into account & 8 & 12.0 & 42 & 88.0 \\
\hline \multicolumn{5}{|l|}{ Observations of symptoms and reactions } \\
\hline Related to course of above disease(s) in general & 22 & 44.0 & 28 & 56.0 \\
\hline Related to course of above disease(s) in patient & 38 & 76.0 & 12 & 24.0 \\
\hline $\begin{array}{l}\text { Related to complications due to therapy ( each medication and each } \\
\text { procedure) }\end{array}$ & 16 & 34.0 & 36 & 68.0 \\
\hline Vital signs & 50 & $\mathbf{1 0 0 . 0}$ & 0 & 0.0 \\
\hline Patient to his condition & 50 & 100.0 & 0 & 0.0 \\
\hline Patient to his course of disease(s) & 38 & 76.0 & 12 & 24.0 \\
\hline \multicolumn{5}{|l|}{ Supervision of patient } \\
\hline Evidence that initial nursing diagnosis was made & 32 & 64.0 & 18 & 36.0 \\
\hline Safety of patient & 48 & 96.0 & 2 & 4.0 \\
\hline Security of patient & 50 & 100.0 & 0 & 0.0 \\
\hline Adaptation (support of patient in reaction to condition and care) & 40 & 80.0 & 10 & 20.0 \\
\hline Continuing assessment of patient's condition and capacity & 6 & 12.0 & 44 & 88.0 \\
\hline Nursing plans changed in accordance with assessment & 36 & 72.0 & 14 & 28.0 \\
\hline Interaction with family and with others considered & 18 & 36.0 & 32 & 64.0 \\
\hline \multicolumn{5}{|l|}{ Supervision of those participating in care ( Except the physician) } \\
\hline Care taught to patient, family, or others, nursing personnel & 18 & 36.0 & 32 & 64.0 \\
\hline Physical , emotional , mental capacity to learn considered & 20 & 40.0 & 30 & 60.0 \\
\hline Continuity of supervision to those taught & 14 & 26.0 & 36 & 74.0 \\
\hline Support of those giving care & 38 & 76.0 & 12 & 24.0 \\
\hline
\end{tabular}


Table(1) conti : Distribution of auditing seven nursing functions at Intensive care Unit in Main Assiut University Hospital.

\begin{tabular}{|c|c|c|c|c|}
\hline \multirow[t]{2}{*}{ Items } & \multicolumn{2}{|c|}{ Yes } & \multicolumn{2}{|c|}{ No } \\
\hline & No. & $\%$ & No. & $\%$ \\
\hline \multicolumn{5}{|l|}{ Reporting and recording } \\
\hline Facts on which further care depended were recorded & 46 & 82.0 & 4 & 8.0 \\
\hline Essential facts reported to physician & 44 & $\mathbf{8 8 . 0}$ & 6 & 12.0 \\
\hline Reporting of facts included evaluation of there & 32 & 64.0 & 18 & 36.0 \\
\hline Patient or family altered as to what to report to physician & 8 & 16.0 & 42 & 84.0 \\
\hline Record permitted continuity of intramural and extramural care & 12 & 24.0 & 36 & 76.0 \\
\hline \multicolumn{5}{|l|}{ Application and execution of nursing procedures and techniques } \\
\hline Administration and/or supervision of medications & 50 & $\mathbf{1 0 0 . 0}$ & 0 & 0.0 \\
\hline Personal care (Bathing, oral hygiene, skin, nail care, shampoo) & 50 & $\mathbf{1 0 0 . 0}$ & 0 & 0.0 \\
\hline Nutrition (including special diets) & 50 & $\mathbf{1 0 0 . 0}$ & 0 & 0.0 \\
\hline Fluid balance plus electrolytes & 40 & 80.0 & 10 & 20.0 \\
\hline Elimination & 50 & $\mathbf{1 0 0 . 0}$ & 0 & 0.0 \\
\hline Rest and sleep & 40 & 80.0 & 10 & 20.0 \\
\hline Physical activity & 30 & 60.0 & 20 & 40.0 \\
\hline Irrigations (including enemas) & 32 & 64.0 & 18 & 36.0 \\
\hline Dressings and bandages & 42 & 88.0 & 8 & 12.0 \\
\hline Formal exercise program & 18 & 36.0 & 32 & 64.0 \\
\hline Rehabilitation (other than formal exercise) & 12 & 24.0 & 38 & 76.0 \\
\hline Prevention of complications and infections & 28 & 56.0 & 22 & 44.0 \\
\hline Recreation, diversion & 32 & 64.0 & 28 & 36.0 \\
\hline Clinical procedures-urine analysis, $\mathrm{B} / \mathrm{P}$ & 14 & 28.0 & 36 & 72.0 \\
\hline $\begin{array}{l}\text { Special treatments (care of tracheotomy, use of oxygen, } \\
\text { colostomy of catheter care, etc) }\end{array}$ & 36 & 72.0 & 14 & 28.0 \\
\hline Procedures and techniques taught to patient & 26 & 52.0 & 24 & 48.0 \\
\hline \multicolumn{5}{|c|}{ Promotion of physical and emotional health by direction and teaching } \\
\hline Plans for medical emergency evident & 38 & 76.0 & 12 & 24.0 \\
\hline Emotional support to patient & 42 & 84.0 & 8 & 16.0 \\
\hline Emotional support to family & 16 & 32.0 & 34 & 68.0 \\
\hline Teaching promotion and maintenance of health & 30 & 60.0 & 20 & 40.0 \\
\hline $\begin{array}{l}\text { Evaluation of need for additional resources (Spiritual, social } \\
\text { service, homemaker service, physical or occupational therapy) }\end{array}$ & 36 & 72.0 & 14 & 28.0 \\
\hline Action taken in regard to needs identified & 38 & 76.0 & 12 & 24.0 \\
\hline
\end{tabular}

Table (2): Mean scores of auditing nursing functions at general Intensive Care Unit in Assiut University Hospital

\begin{tabular}{|c|l|c|}
\hline No. & \multicolumn{1}{|c|}{ Nursing Functions } & Mean \pm SD \\
\hline I- & Application and execution of physician's legal orders. & $29.66 \pm 15.35$ \\
\hline II- & Observations of symptoms and reactions. & $\mathbf{3 5 . 6 6} \pm \mathbf{1 4 . 1 0}$ \\
\hline III- & Supervision of patient. & $\mathbf{3 2 . 8 6} \pm \mathbf{1 5 . 9 5}$ \\
\hline IV- & Supervision of those participating in care (Except the physician). & $22.50 \pm 10.63$ \\
\hline V- & Reporting and recording & $28.40 \pm 17.68$ \\
\hline VI- & Application and execution of nursing procedures and techniques. & $\mathbf{3 4 . 3 7} \pm \mathbf{1 2 . 7 1}$ \\
\hline VII- & Promotion of physical and emotional health by direction and teaching. & $\mathbf{3 3 . 3 3} \pm \mathbf{9 . 3 5}$ \\
\hline
\end{tabular}


Fig. (1): Total score of nursing functions performed by studied nurses at general Intensive Care Unit in main Assiut University Hospital ( $\mathrm{n}=\mathbf{5 0}$ patient records)

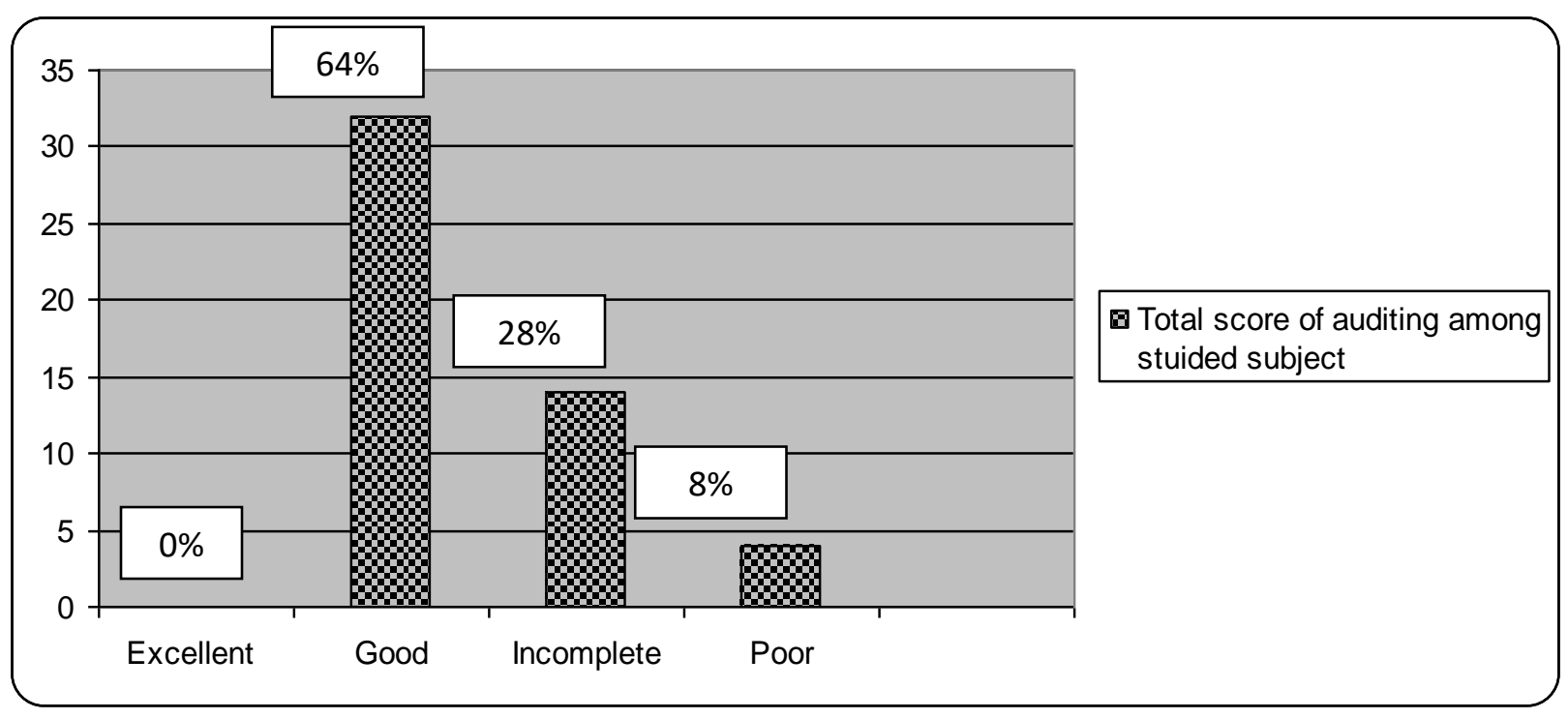

Table (1): Shows that the highest items that performed in application and execution of physician's legal orders factor were medical diagnosis complete , observations of symptoms and reactions and all of the studied subjects auditing vital signs, patient to his condition, and $76 \%$ auditing observations of symptoms and reactions related to course of above disease(s) in patient ; as regard to supervision of patient factor all of them considering security of patient and majority of them auditing safety of patient and adaptation (support of patient in reaction to condition and care) $(96 \%$ and $80 \%$ ) respectively. In addition, more than three quarters support of those giving care in supervision of those participating in care (Except the physician) factor; majority of them auditing of essential facts reported to physician followed by facts on which further care depended were recorded in reporting and recording factor $(88$ $\%$ and $82 \%$ ) respectively. All of them auditing of administration and/or supervision of medications, personal care (bathing, oral hygiene, skin, nail care, shampoo), nutrition (including special diets), and elimination in the application and execution of nursing procedures and techniques factors. Finally, more than three quarters of them auditing of emotional support to patient and Plans for medical emergency evident in the promotion of physical and emotional health by direction and teaching factor.

Table (2): Illustrated that highest mean scores of nursing functions were in observations of symptoms and reactions, application and execution of nursing procedures and techniques, promotion of physical and emotional health by direction and teaching, and supervision of patient $(\mathbf{3 5 . 6 6} \pm \mathbf{1 4 . 1 0} ; \mathbf{3 4 . 3 7} \pm \mathbf{1 2 . 7 1}$; $33.33 \pm 9.35$; and $32.86 \pm 15.95$ ) respectively.

Figure (1): Illustrated that none of the study subject had an excellent auditing of nursing and medical care, nearly two thirds of the data audited were good, more than one quarter were incomplete in auditing of nursing and medical care; and eight percent had a poor auditing of nursing and medical care.

\section{Discussion}

Clinical audit is a process that seeks to improve patient care and outcomes through a systematic review of care against explicit measures and the implementation of change in practice if needed (Dixon, 1996). Clinical audit may be used to measure adherence to evidence-based clinical practice guidelines, and is a useful way for clinicians to measure their current practice and subsequently identify any gaps. The main aim of clinical audit is to rigorously measure how well something is done and to provide feedback to improve local performance of clinical care. Clinical audit has the potential to assure or improve direct patient care, though patients are not the only beneficiaries of the process (Middleton, 1996).

The result of the present study as shown in (table, 1) shows that the highest items that performed in application and execution of physician's legal orders factor were medical diagnosis complete, observations of symptoms and reactions and all of the studied subjects auditing vital signs, patient to his condition, and $76 \%$ auditing related to course of above disease(s) in patient; as regard to supervision of 
patient factor all of them considering security of patient and majority of them auditing safety of patient and adaptation (support of patient in reaction to condition and care) (96\% and 80\% ) respectively. In addition, more than three quarters support of those giving care in supervision of those participating in care (except the physician) factor; majority of them auditing of essential facts reported to physician followed by facts on which further care depended were recorded in reporting and recording factor (88 $\%$ \& $82 \%$ ) respectively. All of them auditing of administration and/or supervision of medications, personal care (bathing, oral hygiene, skin, nail care, shampoo), nutrition (including special diets), and elimination in the application and execution of nursing procedures and techniques factors. Finally, more than three quarters of them auditing of emotional support to patient and Plans for medical emergency evident in the promotion of physical and emotional health by direction and teaching factor. These results supported by Seddon \& Buchanan, (2006) who mentioned that clinical audit identifies any gaps between what is done and what should be done, and rectifies any deficiencies in the actual processes of care. In this article, the steps involved in a clinical audit, how it is different to research, and the question of whether clinical audit requires ethical approval are explored.

The result of the current study illustrated that highest mean scores of nursing functions were in observations of symptoms and reactions, application and execution of nursing procedures and techniques, promotion of physical and emotional health by direction and teaching, and supervision of patient $(35.66 \pm 14.10 ; 34.37 \pm 12.71 ; 33.33 \pm 9.35$; and $32.86 \pm 15.95$ ) respectively as shown in (table, 2$)$. This might be attributed to nurses who working at I.C.U providing direct nursing care by application of all procedures and techniques for critically ill patients. These results is supported by Collis, ( 2006) and Gardner, Gardner \& O'Connell, (2010) who mentioned that an audit is an important tool to provide measurement and feedback on the process and outcome of clinical practice. Audit of the process of care includes measuring service outcomes that are influenced by nurse practitioner service (such as wait times and other specific service key performance indicators).

The result of the present study in (Figure, 1) illustrated that none of the study subject had an excellent auditing of nursing and medical care, nearly two thirds of the data audited were good, more than one quarter were incomplete in auditing of nursing and medical care; and eight percent had a poor auditing of nursing and medical care. This result might be attributed to all patients in I.C.U need for professional nurses that provide intensive care for them, so not all nurses will be excellent in providing care for patients. These results supported by Hearnshaw \& Baker, (1998) who clarified that the level of audit activity in general practice is reasonably high, and most of the audits result in change. The number of audits per practice seems to be independent of the level of funding that the medical audit advisory group has received. Although there is room for improvement in the levels of effective audit activity in general practice, continued support by the professionally led audit groups could enable all practices to undertake effective audit that leads to improvement in patient care.

In addition, Novo, Ridanovic and Maric, (2006) mentioned that clinical audit is used as a term for any kind of audit leaded by professionals in health care, and should not be complicated or unpleasant job. However, the recent focus on clinical governance has resulted in the need for effective methods of systematically reviewing quality, Clinical audit is a tool that has been created for this purpose. Moreover, Clinical audit as Quinn, (1998) prefer patient care audit, is just one tool that is seen as essential in ensuring quality of patient care.

\section{Conclusions}

None of the study subject had an excellent auditing of nursing and medical care, nearly two thirds of the data audited were good, more than one quarter were poor in auditing of nursing and medical care; and eight percent had a poor auditing of nursing and medical care.

\section{Recommendations}

- In- service training program for nurses needed to improve the quality of service.

- Replicate of these study at different units in health care settings to ensure effectiveness of the tool and serving as basis for planning new programs; identification of areas of strength and weakness in various settings.

- Continuous supervision for personnel providing care.

- Further research should be done on application \& execution of physician's orders and documentation.

\section{References}

1. Centre for Clinical Governance Research in Health, (2009): Clinical audit: a comprehensive review of the literature. University of New South Wales (UNSW), Faculty of Medicine, Sydney, NSW 2052, Pp.1-64.

2. Collis, S., (2006): "A., review of the literature on the nurse role in clinical audit." Nursing Times 102(12): Pp. 38-40. 
3. Cowan, P., (2002): "The role of clinical audit in risk reduction." British Journal of Clinical Governance 7(3): Pp. 220-223.

4. Gillies, D., (1984): Nursing Management - A., System Approach. Patient Care Audit. Philadelphia; WB Saunders.

5. Hearnshaw, H., \& Baker, R., (1998): "A survey of audit activity in general practice." British Journal of General Practice 48(427): Pp.979-81.

6. Hysong S., Best R., \& Pugh J., (2006): Audit and feedback and clinical practice guideline adherence: Making feedback actionable. Implementation Science, 1(1): P.9.

7. Majella, D., (2008): Healthcare Audit Criteria and Guidance, OQR014 (2), v1, Pp. 1-43.

8. Nightingale, F., (1859): Notes on hospitals. London : John W. Parker \& Son.

9. Novo A., Ridanovic Z., \& Maric V., (2006): "Clinical audit as method of quality improvement of healthcare in patients with diabetes, stroke and in cesarean section. 60(3): Pp.185-189.

10. Phaneuf's nursing audit (1955), cited by Diana Sale, (2005): Understanding clinical governance and quality assurance, Chapter 12, Pp.206: 212.

11. Reena , J., \& Indarjit, W., (2011): Nursing audit, The Nursing Journal of India, Vol.(CII), No. 6, Pp. 1-3.

12. Seddon, M., \& Buchanan, N., (2006): "Quality improvement in New Zealand healthcare. Part 3: achieving effective care through clinical audit." New Zealand Medical Journal, 119(1239): U2108.

13. Walker, L., (2006): Setting standards for planning off duty \& audit of practice. Nursing Times; 102(21):Pp. 30-32.

14. Schlescelman, J., (1982): Case-control studies. Oxford University Press, New York.

15. Gardner, G., Gardner, A., \& O'Connell, J., (2014): Using the Donabedian framework to examine the quality and safety of nursing service innovation. Journal of Clinical Nursing, 23(12), 145-55. doi:10.1111/jocn.1214. 